\title{
Una contribución al diseño hidráulico de puentes en zonas costeras
}

\author{
J.L. Sánchez-Bribiesca (†) y J. Gracia-Sánchez \\ Instituto de Ingeniería, UNAM \\ E-mail: jgs@pumas.iingen.unam.mx
}

(recibido: noviembre de 2001; aceptado: diciembre de 2002)

\begin{abstract}
Resumen
Se presenta un criterio para determinar el ancho y la altura de puentes sobre ríos de zonas costeras desde el punto de vista hidráulico. El criterio empleó información de puentes fallados y no fallados (sobre una misma corriente); asimismo, permitió tanto el diseño de puentes nuevos, como la revisión de otros existentes. Se incluyen ejemplos numéricos.
\end{abstract}

Descriptores: diseño, hidráulica, puentes, zonas costeras, crecientes, falla.

\begin{abstract}
It is pro posed a cri terion to deter mine, from thehy drau lic point of view, thewidth and the height of bridges of coastal zones. The cri te rion uses in for ma tion of failed and not failed bridges (both in thesamecur rent). Thecri terion al lows the design of new bridges and the revi sion of theex isting ones oth ers ex istent ones. N u mer i cal ex am plesarein duded.
\end{abstract}

Keywords: de sign, hy drau lics, bridges, coastal zones, flood, fail ure.

\section{Introducción}

Los ríos que fluyen en zonas costeras estrechas suelen alojarse en valles relativamente angostos, de baja pendiente, en donde la distancia entre las montañas y la playa no es muy grande y cuyo suelo erosionable está constituido por material granular, que va desde arenas muy finas hasta gravas.

Cuando en estos ríos se presentan crecientes con gastos muy grandes, en atención a que los gastos de material sólido son notoriamente menores que los de agua, el fondo de los cauces prácticamente no se profundiza (Henderson, 1966), por lo cual, para conducir los caudales extraordinarios, los anchos de los ríos y sus tirantes se incrementan considerablemente. Estos fenómenos dan lugar a que se colapsen los puentes colocados en estos ríos si no tienen la longitud y la altura necesarias para resistir el paso de las crecientes. En este trabajo se expone un método que permite determinar cuáles podrían ser esas longitudes y alturas. 


\section{Fundamentos}

\section{A ntecedentes}

En el trabajo de Sánchez et al. (1999a), se describe un método para dimensionar los puentes en las zonas costeras, éste se basó únicamente en consideraciones teóricas, ya que al ser elaborado no se disponía de información sobre el comportamiento de estas estructuras al presentarse una creciente. Este método es muy laborioso y requiere de muchos datos que no siempre están disponibles, por lo que no resulta adecuado para enfrentar situaciones de emergencia, como la reconstrucción rápida de los puentes dañados para reiniciar pronto el tránsito. Por tales motivos, los autores pensaron en desarrollar el método que se describe en este trabajo, teniendo en cuenta los datos de varios puentes que no sufrieron daños y de otros que se colapsaron du rante las crecientes.

Los valles angostos en donde fluyen los ríos de las zonas costeras estrechas y donde la distancia de la falda de las montañas a la orilla del mar no es muy grande, tienen pendientes longitudinales $\mathrm{S}$ que decrecen hacia la costa, pero pueden estimarse en las zonas adyacentes a los puentes mediante planos topográficos con escalas relativamente grandes. Las secciones transversales de estos valles suelen tener pendientes (SV, Figura 1) más o menos simétricas de la zona más baja hacia el límite de los valles.

El ma te rial gran u lar de los terrenos de estos valles suele tener diámetros medios desde $\bar{D}=$ $\pm 0.00005 \mathrm{~m}$ (arena muy fina) y pendiente $\mathrm{S}= \pm$ 0.00001 , hasta $\bar{D}= \pm 0.005 \mathrm{~m}$ (gravas) con pendientes $S= \pm 0.001$. Suponiendo que el coeficiente de rugosidad según Manning depende del tamaño de los granos, en (Chow, 1959) se propone que su valor se calcule con la ecuación

$$
\mathrm{n}=0144 \bar{D}^{0.2}
$$

La ecuación anterior fue calibrada de las observaciones de campo, por ello se empleó en este trabajo, aunque generalmente en la literatura se propone emplear la relación de Strickler:

$$
\mathrm{n}=\operatorname{cte}^{*} \bar{D}^{1 / 6}
$$

Por otra parte, si B es el ancho del río, varios autores Henderson (1966), Lai y Shen (1996) y

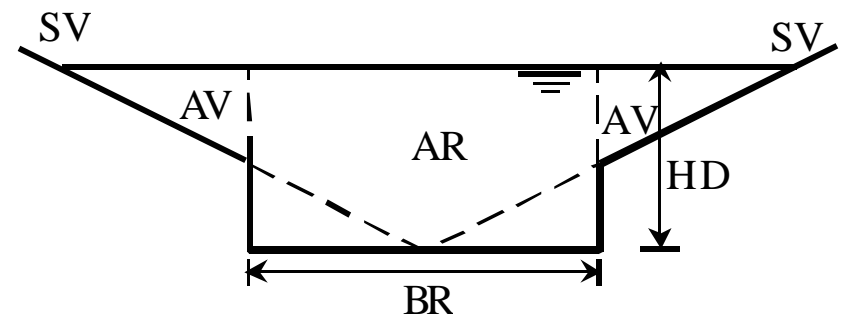

Figura 1. Sección aguas arriba 
White (2001) coinciden en que si $Q$ es el gasto que se conduce, se puede establecer la relación

$$
B=K o Q^{0.5}
$$

Un valor Ko para lechos arenosos es cercano a 4 y, según los datos reportados por Henderson (1966), su magnitud difícilmente sería menor que la mitad o mucho mayor que el doble.

En el trabajo de Sánchez et al. (1999a), se muestran ejemplos de puentes colapsados, en donde puede verse que varias superestructuras se desplazaron aguas abajo, revelando que unas giraron 90 을 hasta orientarse en sentido de la corriente. En adición, se pudo observar que hubo sitios en donde se colocaron dos puentes a corta distancia uno de otro y que el de mayor altura no sufrió daños; sin embargo, el más bajo se destruyó considerablemente, incluyendo una erosión muy fuerte de los terraplenes de acceso. Todo esto conduce a pensar que los desplazamientos de las superestructuras se deben a fuerzas horizontales en el sentido de la corriente, que pueden explicarse si los puentes fueron rebasados, en cuyo caso, también se dañarían los terraplenes de acceso. Además, debe tenerse en cuenta que si los puentes son de corta longitud, al estrecharse el cauce pueden producirse velocidades erosivas que socaven el entorno de las pilas, debilitando su estabilidad.

\section{Funcionamiento de los ríos en la proximidad de los puentes}

Si HP es la altura de un puente (desnivel entre el paño inferior de la superestructura y el fondo del río), BP es su longitud y $Q$ el gasto que circula. Para analizar el funcionamiento del río en la zona del puente se pueden establecer dos parámetros:

$$
X=\operatorname{Ln}\left(Q /\left(\sqrt{g} * B P^{2.5}\right)\right)
$$

(4)

$$
Y=\operatorname{Ln}(H P / B P)
$$

Con estos parámetros y con el trabajo de Sánchez et al. (1999b), se hizo el análisis de 12 puentes en la zona costera de Chiapas, de acuerdo con los datos disponibles. Con ellos se determinó la ecuación de la envolvente que se muestra en la figura 3. Dicha ecuación es

$Y=2.134+1.4628 X+0.1375 X^{2}+0.0055 X^{3}$

Nótese que $X$ e $Y$ relacionan parámetros adimensionales propuestos en este trabajo para representar al fenómeno hidráulico. Se probaron otros números adimensionales, pero los presentados aquí resultaron los mejores. De esta forma, se observó que fallaron los tres puentes, cuyas coordenadas marcadas con cuadros corresponden a puntos situados DEBAJO de la envolvente, en tanto que para los nueve que no sufrieron daños, los puntos se situaron en la envolvente o ARRIBA de ella, esto hace suponer que dicha envolvente representa una situación límite a partir de la cual puede ocurrir el colapso.

Por otro lado, del análisis de los datos registrados en los puentes fallados, se propuso considerar que la velocidad que corresponde a la situación límite depende del gasto y del tamaño de los granos, para que su magnitud sea calculada con la siguiente ecuación obtenida a partir de los datos de campo.

$V P=0.4 Q^{0.15} e^{\left(122533+0.1835(\operatorname{Ln} \bar{D})+0.0209(\operatorname{Ln} \bar{D})^{2}+0.0033(\operatorname{Ln} \bar{D})^{3}\right)}$ 
Una contribución al diseño hidráulico de puentes en zonas costeras

(6)

De acuerdo con estas ideas, se elaboró el programa "DIMPUE.BAS" que permitió determinar para cada combinación de $Q$ y $\bar{D}$ cuáles serían los valores de VP, Ko, S y n, a fin de comprobar la congruencia de los resultados. Este programa esencialmente resuelve la siguiente ecuación para valores de la vari able $\mathrm{x}$ definida como

$$
x=Q /\left(\sqrt{g} * B P^{25}\right) .
$$

$$
V P=\frac{Q}{e^{2.134+1.4628(\operatorname{Lnx})+0.1375(\operatorname{Lnx})^{2}+0.0055(\operatorname{Lnx})^{3}}\left[\left(\frac{Q}{\sqrt{g} x}\right)^{1 / 2.5}\right]^{2}}
$$

(7)

además

$$
B R=\left(\frac{Q}{\sqrt{g} x}\right)^{1 / 2.5}
$$

$$
H=B R e^{2.134+1.4628\left(\operatorname{Lnx} x+0.1375(\operatorname{Ln} x)^{2}+0.0055(\operatorname{Lnx})^{3}\right.}
$$

$$
\begin{aligned}
\mathrm{KO} & =\mathrm{BR} / \sqrt{\mathrm{Q}} \\
S & =\left(\frac{n V P}{H^{2 / 3}}\right)^{2}
\end{aligned}
$$

En la tabla 1 se muestran los datos logrados con este programa para nueve casos analizados. Aquí puede verse una concordancia satisfactoria de los resultados para diferentes valores propuestos de $\bar{D}$, n y $\mathrm{Q}$.

Una vez comprobada la congruencia, se procedió a la elaboración de los programas "REPU03.BAS" y "PRPU01.BAS", el primero para analizar el comportamiento de un puente ya construido y el segundo para diseñar uno nuevo. Para operar estos programas fue necesario hacer las consideraciones que se indican a continuación.

En la figura 1 se muestra la sección transversal de un río aguas abajo de un puente y se indica el ancho del río $B R$ que se establecería al fluir un gasto $\mathrm{Q}$ con un tirante $\mathrm{H}$, teniendo en cuenta el gasto $Q_{R}$ que pasa por la sección AR y el gasto QV por las subsecciones

1 Este programa y los que adelante se presentan están disponibles con los autores.

Tabla 1. Comparación de resultados

\begin{tabular}{lcccc}
\hline & $\mathrm{Q}=300 \mathrm{~m} / \mathrm{s}$ & $\mathrm{Q}=600 \mathrm{~m}^{3} / \mathrm{s}$ & $\mathrm{Q}=1200 \mathrm{~m}^{3} / \mathrm{s}$ & \\
\hline $\bar{D}=0.00005 \mathrm{~m}$ & 4.228 & 5.611 & 7.445 & $\mathrm{H}(\mathrm{m})$ \\
$\mathrm{n}=0.0225$ & 153.06 & 207.72 & 281.70 & $\mathrm{BR}(\mathrm{m})$ \\
& 0.464 & 0.515 & 0.572 & $\mathrm{VP}(\mathrm{m} / \mathrm{s})$ \\
& 8.84 & 8.48 & 8.13 & $\mathrm{KO}$ \\
$\bar{D}=0.0005 \mathrm{~m}$ & 0.0000125 & 0.0000105 & 0.0000089 & $\mathrm{~S}$ \\
$\mathrm{n}=0.03$ & 3.837 & 5.068 & 6.698 & $\mathrm{H}(\mathrm{m})$ \\
& 75.33 & 102.75 & 140.16 & $\mathrm{BR}(\mathrm{m})$ \\
& 1.038 & 1.152 & 1.278 & $\mathrm{VP}(\mathrm{m} / \mathrm{s})$ \\
& 4.35 & 4.20 & 4.05 & $\mathrm{Ko}$ \\
$\bar{D}=0.005$ & 0.000178 & 0.000151 & 0.000128 & $\mathrm{~S}$ \\
$\mathrm{n}=0.05$ & 4.067 & 5.303 & 6.927 & $\mathrm{H}(\mathrm{m})$ \\
& 44.33 & 61.42 & 84.75 & $\mathrm{BR}(\mathrm{m})$ \\
& 1.660 & 1.848 & 2.044 & $\mathrm{VP}(\mathrm{m} / \mathrm{s})$ \\
& 2.56 & 2.51 & 2.45 & $\mathrm{Ko}$ \\
& 0.00106 & 0.000914 & 0.000789 & $\mathrm{~S}$ \\
\hline
\end{tabular}

Nota: $\mathrm{H}$ es el tirante del río de ancho $\mathrm{BR}$ 


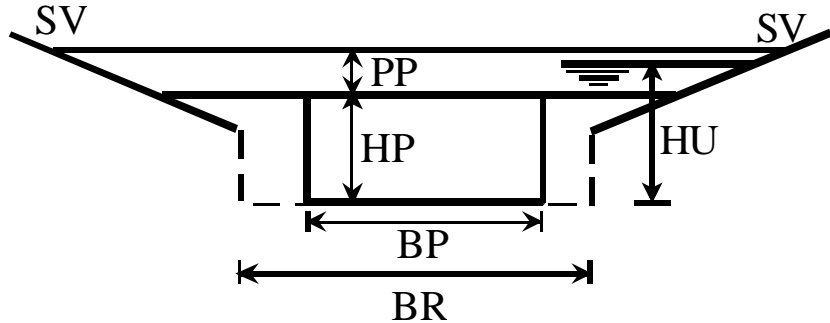

Figura 2. Sección aguas abajo

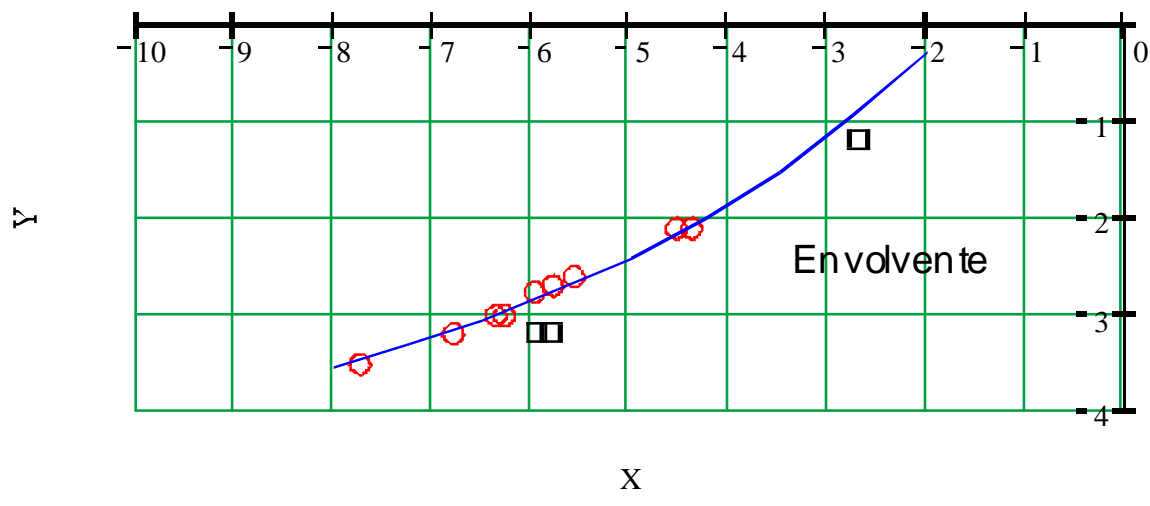

Figura 3. Ajuste de datos

AV y, supuesto que $Q_{R}+Q_{V}=Q$, aguas abajo del puente se presentaría un tirante $\mathrm{H}_{D} \leq \mathrm{H}$.

En la figura 2 se observa la sección transversal de un río aguas arriba de un puente de longitud $\mathrm{BP}$, altura HP y peralte PP. Aquí se indica el tirante HU aguas arriba del puente, que depende de la forma en que éste trabaje.

En la sección del río en donde se encuentra el puente, para el gasto $Q$ se presentó una velocidad VPP distinta de VP, que dependió de la forma de funcionamiento del puente.

Para emplear el programa "REPU03.BAS" es necesario especificar la magnitud de la pendiente transversal SV y los datos del puente, $B P, H P$, y PP, así como el gasto $Q$ de la creciente. Además, se debe indicar la pendiente longitudinalS y estima un valor de diámetro $\bar{D}$ que se comprobará posteriormente.

El programa calculó $n$ con la ecuación 1 y VP con la 6 y mediante el método de aproximaciones sucesivas, previa determinación de $X=\operatorname{Ln}\left(Q /\left(\sqrt{g} * B R^{2.5}\right)\right)$ y $Y X=\operatorname{Ln}(H / B R)$; asimismo, calcula los valores de $H$ y BR, comprobando que con una pequeña tolerancia se satisface la igualdad $\mathrm{VP}=\mathrm{Q} /(\mathrm{BR} * \mathrm{H})$. Enseguida, se estima el tirante HD aguas abajo del puente, de 
acuerdo con los gastos $Q R$ y $Q V$ antes mencionados.

También valua el parámetro $\mathrm{YN}=\mathrm{Ln}(\mathrm{HP} / \mathrm{BR})$.

Para calcular HD que es la variable

$Q=\left.\left[2\left[\left(\frac{H D}{S V}-\frac{B R}{2}\right)\left(\frac{H D-\frac{B R * S V}{2}}{2}\right)\right]\right]{\frac{V P}{H^{2 / 3}}}_{[}^{\left[\left(\frac{H D}{S V}-\frac{B R}{2}\right)^{2}+\left(H D-\frac{B R * S V}{2}\right)\right]^{0.5}+H D-\frac{B R * S V}{2}}\right|^{2 / 3}+B R\left(\frac{V P}{H^{2 / 3}}\right) H D^{5 / 3} \ldots$

previamente definida, se resuelve con la ecuación

Ésta se obtiene a partir de todas las consideraciones antes presentadas y las características geométricas de la figura 1. Entonces, si $H D<H P$, se concluye que el puente funcionará bien; pero si $\mathrm{HD}>\mathrm{HP}$, el programa procede a calcular el tirante aguas arriba, HU, cuando el puente funcione como alcantarilla y que podría no ser rebasado 0 quedar totalmente sumergido si $\mathrm{HU}>(\mathrm{HP}+$ $\mathrm{PP})$, en cuyo caso hace la determinación de $\mathrm{HU}$ con el método de aproximaciones sucesivas. Terminados todos estos cálculos, el programa despliega los valores de todos los parámetros de interés, con los que puede comprobarse el funcionamiento del puente. En cualquier caso, se necesita comprobar que la pendiente lon gitudinal concuerde con la que calcula el programa, concordancia que puede lograrse variando el valor de $\bar{D}$.

Para utilizar el programa "PRPU01.BAS" se requiere indicar las pendientes $S$ y SV, así como el gasto $Q$ de la creciente. Además, se introduce un valor estimado del diámetro $\bar{D}$ que debe comprobarse después. Con el fin de limitar la longitud del puente, también se necesita especificar un coeficiente de reducción $\mathrm{CR}<1$.
Como en el caso anterior, el programa calcula $n, V P, H, B R$ y HD. Después hace BP = $C R * B R$ y se procede a calcular la velocidad VPP y el tirante HPP en la sección del puente, para posteriormente determinar

$$
\begin{gathered}
H P=1.15 * H P P, \quad Y P=\operatorname{Ln}(H P / B P) \\
\text { y } \quad X N=\operatorname{Ln}\left(Q /\left(\sqrt{g} * B P^{2.5}\right)\right)
\end{gathered}
$$

HPP se calcula con la siguiente ecuación

$$
\begin{gathered}
H P P+\frac{\left(\frac{Q}{B R^{*} C R^{*} H P P}\right)^{2}}{2 g}=H D+\frac{V P^{2}}{2 g}+ \\
\frac{\left(V P-\frac{Q}{B R^{*} C R^{*} H P P}\right)^{2}}{2 g}
\end{gathered}
$$

Si resulta que VPP / VP > 1.25, despliega el aviso "REVISA", que implicará aumentar el valor de CR para evitar velocidades erosivas. En cambio, si VPP / VP < 1.25, el programa despliega los valores de los parámetros de interés para comprobar el funcionamiento satisfactorio del puente de dimensiones BP y HP. También en este caso deberá comprobarse 
la concordancia del valor estimado de $\mathrm{S}$ con el que despliega el programa.

Es conveniente señalar que no es indispensable que el lector disponga de los programas señalados en este trabajo, ya que con alguna hoja de cálculo (Mathcad, Matlab, etc.) se pueden realizar los cálculos fácilmente.

\section{Metodología}

Según se indicó en el capítulo anterior, para analizar el funcionamiento de un puente ya construido, cuando se presente una creciente de gasto Q, se usará el programa "REPU03.BAS", al que se le suministrarán los datos que allí se indican. Si el puente funciona correctamente aparecerá el letrero "PUELIB" y se desplegarán los datos HP, HD, LB (libre bordo), S, VP, VPP, X, YX y YP, que permitirán comprobar el funcionamiento satisfactorio.

Si el puente funcionara como alcantarilla, pero sin ser rebasado, aparecerá el letrero "PAHTLI", así como los valores de los parámetros HU, HP, HD, S, VP, VPP, X, YX, y YP, que permitirán hacer la comprobación correspondiente. Otro tanto sucederá si el puente quedara rebasado, en cuyo caso aparecerá el letrero "PAHTAH".

Para diseñar un nuevo puente, se usará el programa "PRPU01.BAS", al cual se le suministrarán los datos mostrados en el capítulo anterior. Se ensayarán valores de CR hasta lograr que no aparezca el letrero "REVISA". Cuando esto suceda el programa desplegará los valores de BP, HP, LB, S, VP, VPP, XN y YN que permitirán comprobar el buen funcionamiento del puente proyectado.

\section{Ejemplo numérico}

\section{Enunciado}

En un río de pendiente longitudinalS $=0.0002$ y transversal $S V=0.05$, se encuentra un puente de dimensiones $\mathrm{BP}=75 \mathrm{~m}, \mathrm{HP}=5 \mathrm{~m}$ y $\mathrm{PP}=1 \mathrm{~m}$ y se desea saber cómo funcionaría si se presentaran crecientes de gastos $Q=400,800$ y $1200 \mathrm{~m}^{3} / \mathrm{s}$. En el caso de que el puente no trabajara satisfactoriamente para alguno de estos gastos, se requiere saber cuáles serían las dimensiones para lograr un buen funcionamiento.

\section{Solución}

Se omiten los tanteos para seleccionar el valor $\bar{D}$. Recuérdese que en cualquier caso es necesario comprobar que la pendiente longitudi nal concuerde con la que calcula el programa, concordancia que puede lograrse variando $\bar{D}$.

A nálisis del puente construido

Tabla 2. Resultados de las simulaciones

\begin{tabular}{cccccccccc}
\hline \multicolumn{10}{c}{ Para Q $=400 \mathrm{~m}^{3} / \mathrm{s}, \bar{D}=0.0006$, PUELIB } \\
\hline HP & HD & LB & HU & S & VP & VPP & X & YX & YP \\
5 & 4.075 & 0.925 & - & 0.000197 & 1.136 & 1.309 & -6.16 & -2.95 & -2.80 \\
\hline \multicolumn{10}{c}{ Para Q $=800 \mathrm{~m} / \mathrm{s}, \bar{D}=0.00075$, PAHTLI } \\
\hline HP & HD & LB & HU & $\mathrm{S}$ & VP & VPP & X & YX & YP \\
5 & 6.307 & - & 5.720 & 0.000204 & 1.332 & 2.133 & -6.11 & -2.93 & -3.05 \\
\hline & & & & & & & & continúa...
\end{tabular}


Los resultados obtenidos con el programa

\begin{tabular}{cccccccccc}
\hline \multicolumn{10}{c}{ Para $\mathrm{Q}=1200 \mathrm{~m} 3 / \mathrm{s}, \bar{D}=0.0008$, PAHTAH } \\
\hline HP & HD & LB & HU & S & VP & VPP & X & YX & YP \\
5 & 6.307 & - & 7.119 & 0.000197 & 1.437 & 3.192 & -6.12 & -2.93 & -3.22 \\
\hline
\end{tabular}

"REPU03.BAS" se muestran en la tabla 2.

Tabla 2. Resultados de las simulaciones (continuación)

Se observó que para $\mathrm{Q}=400 \mathrm{~m} 3 / \mathrm{s}$ el puente funcionaría bien con un libre bordo suficiente, sin que la velocidad en la sección del puente se incrementara notoriamente, de tal manera que el punto de coordenadas X, YP estaría situado arriba de la envolvente mostrada en la figura 3.

Para $Q=800 \mathrm{~m}^{3} / \mathrm{s}$ el puente ya trabajaría como alcantarilla, por lo que habría fuerzas horizontales que tenderían a desplazar a la superestructura hacia aguas abajo y la velocidad en la sección del puente podría ser erosiva. El punto de coordenadas X, YP ahora quedaría situado debajo de la envolvente.

Finalmente, para $\mathrm{Q}=1200 \mathrm{~m}^{3} / \mathrm{s}$, la situación sería todavía peor, porque el puente sería totalmente rebasado con un empuje hor $\mathrm{i}$ zontal muy fuerte sobre la superestructura, la velocidad en la sección del puente aumentaría de modo considerable y habría riesgo de que se erosionaran fuertemente los terraplenes de acceso, y el punto de cooordenadas X, YP quedaría situado todavía más abajo de la envolvente.

Así, puede concluirse que el puente de longitud $B P=75 \mathrm{~m}$ y altura $\mathrm{HP}=5 \mathrm{~m}$, difícilmente podría soportar una creciente de gasto $Q=800 \mathrm{~m}^{3} / \mathrm{s}$ y que para $\mathrm{Q}=1200 \mathrm{~m}^{3} / \mathrm{s}$ podría esperarse prácticamente su destrucción total.

\section{Diseño de nuevos puentes}

Omitiendo los tanteos para seleccionar el valor CR y con el programa "PRPU01.BAS" se obtuvieron los datos que se muestran en la tabla 3.

Aquí se revela que para $Q=800 \mathrm{~m}^{3} / \mathrm{s}$ convendría construir un puente de longitud $\mathrm{BP}$ $=92 \mathrm{~m}$ y altura $\mathrm{HP}=6.10 \mathrm{~m}$, con lo cual no se incrementaría notoriamente la velocidad en la sección del puente, se tendría un libre bordo de $0.8 \mathrm{~m}$ y el punto de coordenadas $\mathrm{XN}, \mathrm{YN}$ quedaría situado arriba de la envolvente.

Finalmente, para $Q=1200 \mathrm{~m}^{3} / \mathrm{s}$ el puente debería tener una longitud $\mathrm{BP}=109 \mathrm{~m}$ y una altura $\mathrm{HP}=7.20 \mathrm{~m}$, de manera que habría un libre bordo de $\pm 0.94 \mathrm{~m}$, no aumentaría notoriamente la velocidad en la sección del puente y el punto de coordenadas XN, YN también quedaría situado arriba de la envolvente.

\section{Conclusiones}

Independientemente de los refinamientos que podrían introducirse en los programas "REPU03.BAS" y "PRPU01.BAS" para lograr una 
J.L. Sánchez-Bribiesca (†) y J. Gracia-Sánchez

Tabla 3. Resultados de las simulaciones

\begin{tabular}{cccccccc}
\hline \multicolumn{7}{c}{ Para Q $=800 \mathrm{~m} 3 / \mathrm{s}, \bar{D}=0.00075, C R=0.87$} \\
\hline BP & HP & LB & S & VP & VPP & XN & YX \\
92.11 & 6.11 & 0.797 & 0.000204 & 1.332 & 1.634 & -5.76 & -2.71 \\
\hline \multicolumn{7}{c}{ Para Q $=1200 \mathrm{~m}^{3} / \mathrm{s}, \bar{D}=0.0008, \mathrm{CR}=0.87$} \\
\hline BP & HP & LB & $\mathrm{S}$ & VP & VPP & XN & YX \\
108.82 & 7.20 & 0.939 & 0.000197 & 1.437 & 1.761 & -5.78 & -2.72 \\
\hline
\end{tabular}

mejor representación de las secciones transversales de los ríos, o una valuación más precisa de las velocidades VP y VPP, los resultados que se muestran en el ejemplo numérico son congruentes entre sí y muestran una concordancia aceptable con los datos de los puentes en donde se ha recabado información.

Como además los programas son de fácil manejo y rápida ejecución, los autores del método propuesto creen que esta metodología podría ser ya una guía para los ingenieros que confronten problemas como los resueltos en el capítulo anterior.

\section{Simbología}

AV Área de triángulo en $\mathrm{m}^{2}$ (figura 1).

AR Área de la sección central del río en $\mathrm{m}^{2}$ (figura 1 ).

B Ancho del río en $m$.

BP Longitud efectiva del puente en $m$ (figura 2).

BR Ancho del río (en $\mathrm{m}$ ) para el tirante $\mathrm{H}$ (figuras 1 y 2 ).

CR Coeficiente de reducción (adimensional, $<1$ ).

$\bar{D} \quad$ Diámetro medio del material sólido del lecho en m.

g Aceleración de la gravedad en $\mathrm{m} / \mathrm{s}^{2}$.
H Tirante en $\mathrm{m}$.

HD Tirante aguas abajo del puente en $\mathrm{m}$ (figuras 1 y 2).

HP Altura del puente en $m$ (figura 1).

HPP Tirante en la sección del puente en $\mathrm{m}$.

HU Tirante aguas arriba del puente en $\mathrm{m}$.

LB Libre bordo en $\mathrm{m}$.

Ko Constante.

$\mathrm{n} \quad$ Coeficiente de rugosidad según Manning ( $\left.\mathrm{s} / \mathrm{m}^{1 / 3}\right)$.

$S$ Pendiente (adimensional).

SV Pendiente de la sección transversal (adimensional, figuras 1 y 2 ).

PP Peralte del puente en $\mathrm{m}$ (figuras 2).

Q Gasto en $\mathrm{m}^{3} / \mathrm{s}$.

QR Gasto por la sección AR en $m^{3} / \mathrm{s}$.

QV Gasto por las secciones AV en $\mathrm{m}^{3} / \mathrm{s}$.

VP Velocidad límite en $\mathrm{m} / \mathrm{s}$.

VPP Velocidad en el puente $\mathrm{m} / \mathrm{s}$.

$X, X N$ Parámetros (adimensionales).

Y, YN, YP, YX Parámetros (adimensionales).

\section{Referencias}

Chow V.T. (1959). O pen Channel H ydraulics. McGraw Hill, New York.

Henderson F.M. (1966). O pen Channel Flow. MacMillan Co., New York. 
Lai J. y Shen H. (1996). Flushing Sediment Through Reservoirs. Journal of Hydraulic Research, Vol. 34, No. 2, 237 - 255.

Sánchez-Bribiesca J.L. et al. (1999a). M odelo matemático para determinar las longitudes y las rasantes de los puentes colocados en los ríos de las franjas costeras. Series de Instituto de Ingeniería, C1-7, México.

Sánchez-Bribiesca J.L. et al. (1999b). Revisión de gastos y capacidad de puentes y alcantarillas, en la región costera del estado de Chiapas (Revisión hidráulica de los puentes en los ríos: "Pijijiapan, "M argaritas" y "Las Hermanas". Informe elaborado para la CNA, México. White R. (2001). Evacuation of Sediments From Reservoirs. Thomas Telford Publishing, H.R. Wallingford, London.

\section{Semblanza de los autores}

José Luis Sánchez-Bribiesca † (1927-2002). Ingeniero civil egresado de la Facultad de Ingeniería, UNAM en 1952 y maestro en ingeniería hidráulica graduado en la misma universidad en 1964. Investigador Emérito del Instituto de Ingeniería, UNAM.

Jesús G racia-Sánchez. Ingeniero civil egresado de la Facultad de Ingeniería, UNAM en 1974, realizó la maestría en 1981 y el doctorado en 1988 en ingeniería hidráulica en la misma Universidad. Actualmente es investigador del Instituto de Ingeniería, UNAM. 